\title{
Clinical Events End Relative to Reference Time Point
}

National Cancer Institute

\section{Source}

National Cancer Institute. Clinical Events End Relative to Reference Time Point. NCI

Thesaurus. Code C87848.

An indicator of the conclusion of a clinical event in relation to a specific point in time. 